\title{
Foresighted Control of Active Foot Prostheses
}

\author{
Kleiner, Bernhard \\ Cesmeci, Denis \\ Fraunhofer Institute for Manufacturing Engineering \\ and Automation IPA \\ Nobelstrasse 12 \\ 70569 Stuttgart | Germany
}

\begin{abstract}
For a better ground adaptation in walking and because of the still unsolved problem of not being able to prevent stumbling accidents with leg prostheses, there is a need to develop a system with a foresighted adaptation of the artificial limb to the ground. An optical measuring system is described which during the ongoing stance phase continually records the three dimensions of the ground in the target area of the next step. From the obtained three dimensional measuring data surrounding bodies or structures, like stairs or ramps, are detected. On the basis of this evaluation, the prosthetic control chooses the optimal adjustment of the prosthetic joint for walking the terrain in the following gait cycle.
\end{abstract}

\section{Introduction}

The state of the art foot and knee prosthesis systems are optimized units and designed for specific walking speed on even ground. Hereby passive spring-damper settings have a defined characteristic curve for the rotation around the idealized ankle for dorsi- and plantar flexion [1]. Thus, a prosthesis foot must be aligned and adjusted accurately for the amputee in order to be able to derive an appropriate revolution characteristic [2]. If an amputee is walking on inclinations, the prosthesis foot will not adapt to the underground due to its non adjustable characteristic. This causes a compensatory flexion mechanism in knee and hip which reduces the velocity of the amputee and affects uncertain movement behaviour [3]. Hydraulic adapted joint systems try to overcome this problem. Such systems exhibit their limitation due their passive adjustment ability [4]. Constant progress in the automation industry in the area of the sensor system and actuating elements encouraged the development of actively steered prostheses feet which are already at the market available or still the subject of the research. Common in all approaches is a micro controller which collects the sensor data and generates a quantitative characteristic of the present gait phase and mode. The motion behaviour generation algorithm on the controller decides on the basis of proprioceptive sensors. This allows only a simultaneous or post-adjustment of the joint to the terrain. After several steps an adjustment can take place to the underground. Therefore the prosthesis foot remains still adjusted to the wrong angle of inclination in the simplest case after committing an inclined level. A delayed adjustment takes effect in a negative way if for example an unexpected doorsill appeared which is not recognized by the adaptive sensor system. The danger for stumbling and falling is not reduced. With the actual state of the art, a step to step adaption with a microprocessor controlled active joint is not possible. The advantage of our proposed system has a significant impact on this.

\section{Approach}

Our approach describes a measurement system as the basis for forward-looking adaptation of prosthetic joints. A two-dimensional laser scanner and an inertial navigation system (INS) are combined to measure the distance to the structure in a two dimensional plain and to estimate the current three dimensional position and orientation of the prosthesis (see Figure 1). The sensors are mounted between ankle and knee joint. During the gait, the laser-scanner is moved mainly around its pitch axis. This enables an observation of a volume in front of the prosthesis. The estimated motion is now used to translate the observed structure in a global coordinate system. We use object detection algorithm for three dimensional point cloud data to detect the occurrence and dimensions of stairs, ramps and holes and other obstacles from the gathered 3D structure of the forward looking terrain. This enables the prosthesis control to use the information from the exteroceptive sensor system for the foresighted adaption of the knee and ankle to the terrain. In comparison to the recent adaption methods a step to step adjustment of the prosthesis can now be realized. 


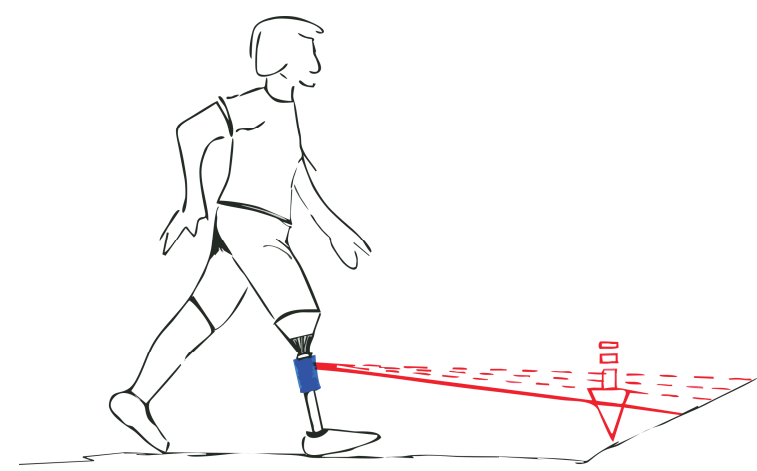

Figure 1: Approach of the measurement system

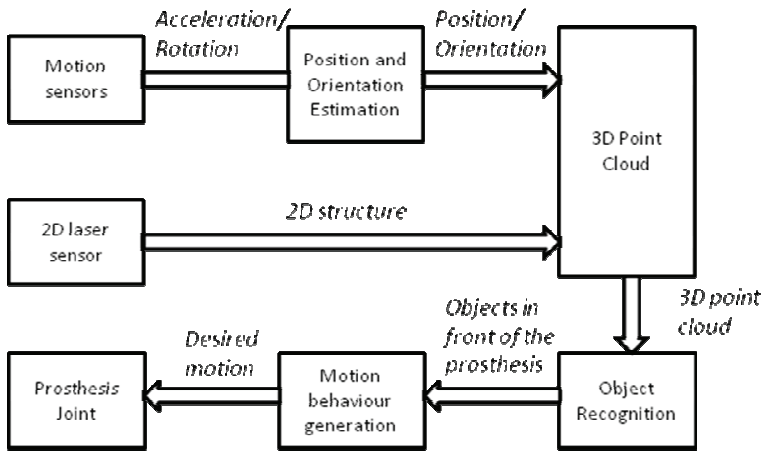

Figure 2: Terrain detection architecture

\subsection{Sensor System}

The distance to the environment is detected with a two dimensional laser scanner [5] which operates on the principle of time of flight. The laser scanner is mounted above the knee (see Figure 3) prosthesis and covers a horizontal sector of $80^{\circ}$ and a distance of around $20 \mathrm{~m}$. An inertial navigation system is mechanically connected to the prosthesis and calculates time-synchronous position and orientation. The inertial navigation system is a result of many years of research at the Fraunhofer Institute IPA [6] in the field of non-referential movement detection using accelerometers and gyroscopes. The three dimensional orientation and position of a body are calculated relative to a reference system with sensor fusion filters. To this end a sensor system is presented which enables the capturing of threedimensional structure during the gait movement.

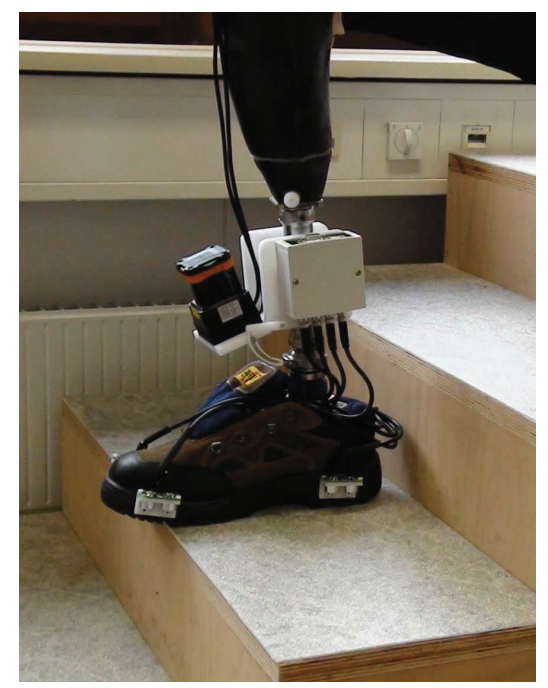

Figure 3: Experimental sensor system for the generation of measurements

\subsection{Terrain detection}

Our method filters the 3D measurement data with a statistical analysis of the measurement point densities. Individual points of the point cloud which may not correspond to a real object are removed from the data set of 3D points and not be considered in further processing steps. The goal is the recognition of a set of objects $M=\{$ Stairs, inclined planes, steps $\}$. These objects are present in most surroundings. Existing object detection methods for point clouds are state of the art in robotic and augmented realty applications. In [7], a method for detection of objects directly from point cloud is presented. For this method, the objects are correlated to the exact shape of the object. This allows no varying in geometry. Instead we use methods to cluster the scene to geometric primitives like triangles [8][9][10]. Furthermore the triangles are clustered to three dimensional plains. An object from the set $\mathrm{M}$ can be represented as a set of plains. Our actual focus in this research is the usage of scalable best fit methods for the modelling of the scene. Furthermore the methods are optimized for the real time processing on an embedded system. 
We use a simulation environment to analyze the clustering and object recognition methods. The simulation software is separated in three modules: (1) The generation of the trajectory of the prosthesis, (2) the generation if the theoretical measurement from the simulated two dimensional laser scanner (3) the simulation of the laserscanner and inertial motion measurement with errors in the system model and Gaussian measurement uncertainties. We validated our concept using the simulation built-up. An example of a recognized ramp is shown in

Figure 4)

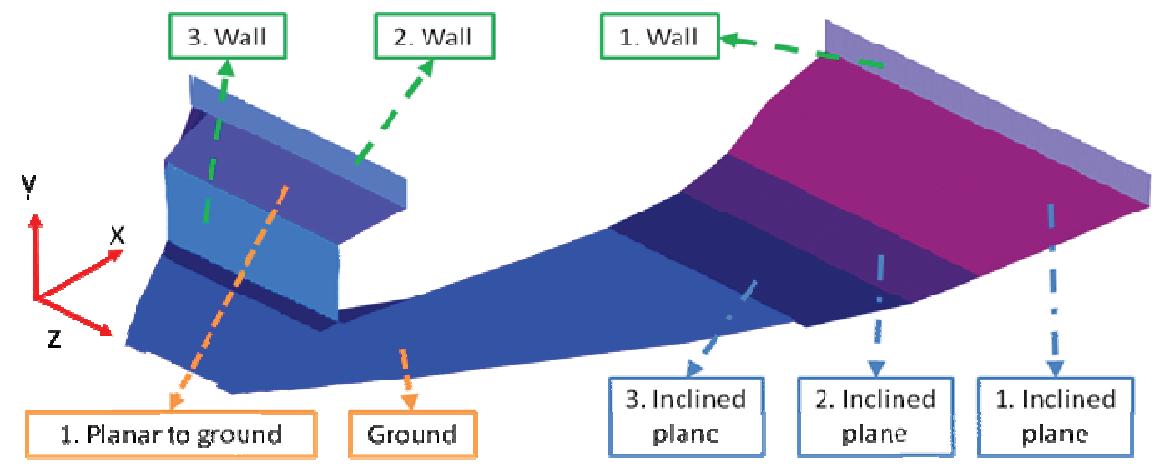

Figure 4: Simulation results for the recognition of plains

\section{$4 \quad$ Measurements and Results}

For the first measurements, we used a simplified the mechanical model of a lower limb. Only a rotation around one axis of the ankle is possible. The measured environment consists of a ramp (see Figure 5) with $6 \%$ inclination and stairs. The data of the terrain detection shows the surface features of the real background and ramp with railing (see Figure 6 ). The result is a match of $93 \%$ in the calculation of the ramp slope and a match of $99 \%$ in calculating the distance from the ramp to the prosthesis. The parameters of the stairs are $15 \mathrm{~cm}$ step height and $30 \mathrm{~cm}$ width and $120 \mathrm{~cm}$ distance to the prosthesis. The result with the terrain detection system is step height of $14.8 \mathrm{~cm}$, a step width of $29.9 \mathrm{~cm}$ and a distance of $120.1 \mathrm{~cm}$. Furthermore, the influence of external light sources on the accuracy of the laser scanner was investigated. We measured a standard deviation of $0.5 \mathrm{~cm}$ with artificial lighting and $1.0 \mathrm{~cm}$ to $1.5 \mathrm{~cm}$ (depending on the material of the ground) with direct alignment to the sunlight.

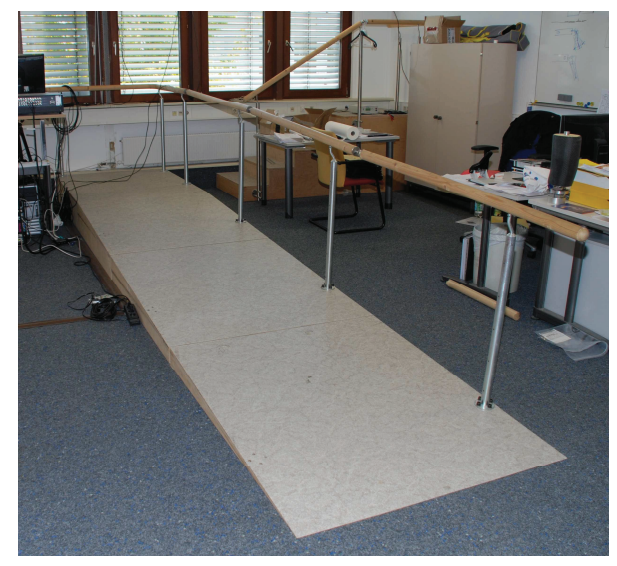

Figure 5: Measurement set in the laboratory

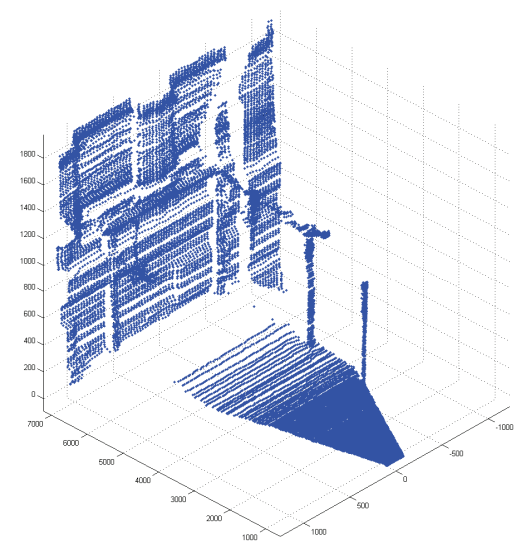

Figure 6: Results for the ramp in the laboratory 
A foresighted adjustment increases the safety for the amputees and lead to a more natural gait on a possible step-by-step adjustment of the prosthetic foot in dorsi flexion and plantar flexion. The described sensors can be used for lower and upper leg prostheses and can be integrated as a separate module. An important issue is the acceptance of the system for the patient. The use of optical sensors for obstacle detection reduces the practical applicability in different weather conditions. An integration of the system in an attractive denture look is part of current research. Further support for the laser measurement system is currently using an ultrasonic measuring system, consisting of four measuring sensors. It continuously captures the distance of the foot to the terrain. A plain can be modelled with the known foot angle and the measured distances.

\section{$6 \quad$ References}

[1] Versluys, R.; Beyl, P.; van Damme, M.; Desomer, A.; van Ham, R. \& Lefeber, D. (2009). Prosthetic feet: state-of-the-art review and the importance of mimicking human ankle-foot biomechanics, Disability and rehabilitation. Assistive technology $4: 65-75$.

[2] Moser, D.; Abimosleh, F.; Zahedi, S.; Harris, G.; Ross, J. \& McCarthy, J. (2009). Biomechanical analysis of a novel automatically self aligning ankle-foot prosthesis, Orthopädie-Technik Quarterly, English edition III.

[3] Fradet, L.; Alimusaj, M.; Braatz, F. \& Wolf, S. I. (2010). Biomechanical analysis of ramp ambulation of transtibial amputees with an adaptive ankle foot system., Gait Posture 32 : 191198.

[4] Sowell, T. T. (1981). Apreliminary clinical evaluation of the Mauch hydraulic foot-ankle system, Prosthetics and orthotics international $5: 87-91$.

[5] Hokuyo (2010). Laserscanner UTM 30LX, http://www.hokuyo-aut.jp/.

[6] Fraunhofer_IPA (2009). Inertiales 3D Ganganalysesystem mit Bewegungsmessung, , http://www.ipa.fraunhofer.de/fileadmin/www.ipa.fhg.de/pdf/Orthopaedie/300_323_InertialeGanga nalyse_D.pdf.

[7] Bennamoun, M.; Mamic, G.J.; Object Recognition Fundamentals and Case Studies. Advances in Pattern Recognition XIII, 2002 Springer, Berlin ISBN 1-85233-398-7.

[8] M. de Berg, M. van Kreveld, M. Overmars and O. Schwarzkopf. Computational Geometry: Algorithms and Applications, Springer-press second edition, Berlin (1998).

[9] H. Woo, E. Kang and S.Y. Wang, A new segmentation method for point cloud data, Int J Mach Tool Manufact 42 (2002), pp. 167-178.

[10] Remondino, F., From point cloud to surface: the modeling and visualization problem, International Archives of Photogrammetry, Remote Sensing and Spatial Information Sciences, Vol. XXXIV. ISPRS International Workshop on "Visualization and Animation of Reality-based 3D Models", 24-28 February 2003, Tarasp-Vulpera, Switzerland 